\title{
BIOARQUEOLOGÍA Y CRONOLOGÍA DEL SITIO NEGRO MUERTO 2 (NORESTE DE PATAGONIA)
}

\author{
ALEJANDRO SERNA* Y LUCIANO PRATES*"
}

\begin{abstract}
RESUMEN
En este trabajo se presentan los primeros resultados del estudio de los restos humanos recuperados en el sitio Negro Muerto 2 (NM2), ubicado en el sector sur de la cuenca media del río Negro (provincia de Río Negro). Durante las actividades de rescate efectuadas en el sitio se recuperaron cuatro individuos: los individuos 1 y 2 formaban parte de un entierro doble (el último sufrió una desarticulación al nivel del cráneo, el cual se dispuso y enterró junto con el primero); y los individuos 3 y 4 , probablemente fueron entierros simples aunque su alto grado de desarticulación no permite confirmarlo. Para el sitio se obtuvieron tres fechados radiocarbónicos de entre ca. 2.000 y 1.500 años AP. El cráneo del individuo 2, fechado en 1.586 \pm 47 años AP, presenta una deformación artificial del tipo tabular oblicua; en este caso la edad obtenida es menor que la esperada para dicha morfología.
\end{abstract}

PALABRAS CLAVE: bioarqueología, Holoceno tardío, Noreste Patagonia, entierros múltiples.

\section{BIOARCHAEOLOGY AND CHRONOLOGY OF THE NEGRO MUERTO 2 SITE (NORTHEAST PATAGONIA)}

\begin{abstract}
In this paper we present the first results for the analysis of human remains recovered at the Negro Muerto 2 (NM2) site, located in the south sector of the Negro river's middle basin (Río Negro Province). During the rescue activities done at the site, four individuals had been recovered: individuals 1 and 2 were part of a double burial (the latter suffered a disarticulation at the level of the skull, which was relocated next to the first one); while individuals 3 and 4 were probably simple burials, although their high degree of disarticulation would not allow us to confirm this. Three radiocarbon dates were obtained for this site between 2,000 and 1,500 years BP. The skull of the individual 2, dated in 1,586 \pm 47 years BP, presents a tabular oblique cranial deformation; in this case the date obtained is less than the expected for such morphology.
\end{abstract}

KEY WORDS: Bioarchaeology, Late Holocene, Northeast Patagonia, multiple burials.

Museo de La Plata, Universidad Nacional de La Plata. Paseo del Bosque s/n (1900), La Plata, Argentina. alejandroserna@ hotmail.com.ar.

* CONICET - Museo de La Plata, Universidad Nacional de La Plata. Paseo del Bosque s/n (1900), La Plata, Argentina. lprates@ fcnym.unlp.edu.ar. 


\section{INTRODUCCIÓN}

El noreste de Patagonia presenta una alta densidad de enterratorios humanos, particularmente en el litoral marítimo y en el sector inferior de la cuenca de los ríos Negro y Colorado. Se reconoce también una importante variabilidad en las modalidades inhumatorias: primarias y secundarias, simples y múltiples, osarios, con presencia de ajuares funerarios y coloración sobre las piezas óseas (Strobel 1867; Moreno 1874; Verneau 1903; Vignati 1923, 1931, 1937; Lehmann-Nitsche 1926, 1930; Fisher y Nacuzzi 1992; Martínez y Figuerero Torres 2000; Martínez et al. 2006; Prates et al. 2010a; Prates et al. 2011, entre otros). Los primeros registros proceden de la laguna del Juncal, en la cuenca del río Negro, y fueron dados a conocer por Strobel (1867, 1968). Casi contemporáneamente, Musters (1997[1869-70]) registra numerosas áreas de entierro en la costa norte del mismo río, a ca. $110 \mathrm{~km}$ de su desembocadura. Luego Moreno (1874) excavó numerosos entierros primarios y secundarios, también cerca de la laguna del Juncal. Lista (1879, 1880), Verneau y de la Vaulx (1900) y Verneau (1903) publicaron y sintetizaron hallazgos similares. Ya en el siglo XX, varios entierros fueron descriptos en la cuenca inferior del río Negro (e.g. Bórmida 1950; Moldes de Extraigas 1983; Fisher y Nacuzzi 1992) y el litoral adyacente (Outes 1926; Vignati 1931, 1937; Sanguinetti de Bórmida 1999), muchos de ellos con presencia de paquetes funerarios, a veces pintados de rojo.

Desde mediados del siglo XIX hasta fines del $\mathrm{XX}$, la mayor parte de los trabajos se enfocaron en la descripción general de los restos, sobre todo de la morfología de los cráneos y de aquellos con evidencias de pintura roja; y sólo se disponía de información detallada en trabajos orientados al estudio de colecciones (Colantonio y Marcelino 1982, 1983; Cocilovo y Guichón 1994; Perez 2006a; Gordon y Ghidini 2007; Bernal et al. 2008; entre otros). Recién a partir de los últimos años, se comenzaron a publicar trabajos con información contextual y/o cronológica precisa de sitios mortuorios (e. g. Martínez et al. 2006; Prates et al. 2010 a y b; Prates et al. 2011). En el caso de la cuenca del río Negro, se recuperaron varios entierros primarios de entre ca. 2.000 y 3.000 años AP en el sitio Loma de los Muertos (Prates et al. 2010 a y b) y un entierro primario doble de ca. 800 años AP en La Victoria 5 (Prates et al. 2011).

En este trabajo se presentan los primeros resultados del análisis de los restos humanos recuperados en el sitio arqueológico Negro Muerto 2 (provincia de Río Negro). El hallazgo del sitio se produjo cuando el personal del establecimiento Negro Muerto expuso varios huesos humanos durante la nivelación del terreno. La suspensión de las tareas al momento de efectuarse los hallazgos y la posterior denuncia al Museo Municipal de Lamarque, permitieron la organización de excavaciones de rescate en el sitio, las cuales fueron ejecutadas en marzo de 2011. La finalidad de este trabajo es aportar información detallada sobre las características principales del contexto mortuorio, como cronología, completitud $e$ integridad general de los esqueletos y modalidades inhumatorias. Se estimaron el sexo y la edad de los individuos, además de establecerse la presencia o ausencia de deformación artificial en los cráneos a partir de la aplicación de técnicas de morfometría geométrica y análisis multivariados.

\section{DESCRIPCIÓN GENERAL DEL SITIO}

El sitio Negro Muerto 2 (NM2) se encuentra en el sector norte del valle medio del río Negro, a ca. $1.500 \mathrm{~m}$ del cauce actual y a ca. $255 \mathrm{~km}$ en línea recta de su desembocadura en el océano Atlántico (3949'0.40"S; 65¹7'2.40”O) (Fig. 1). En este punto el río corre por un área extensa de valle, con un ancho cercano a los $20 \mathrm{~km}$ (medidos entre los límites del valle y las planicies ubicadas al norte y al sur). El paisaje se caracteriza por el predominio de una estepa arbustiva algo más densa y diversa que en los sectores aledaños de planicie, con una altura media del estrato superior de $2 \mathrm{~m}$ y con una mezcla de elementos de las provincias fitogeográficas del Monte y el Espinal (Hauman Merck 1913; Cabrera 1976). Sin embargo, este ambiente perdió completamente su fisonomía debido al desmonte y nivelación realizados en los últimos años, uno de cuyos efectos fue la exposición de los restos del sitio. Geomorfológicamente el sitio se encuentra en la primera terraza de inundación del río (Luchsinger 2006), en la parte interna del meandro de un paleocauce. Antes de la construcción de las represas sobre los colectores cordilleranos del río, estos paleocauces se activaban durante las crecidas estacionales (Prates 2008). En la 


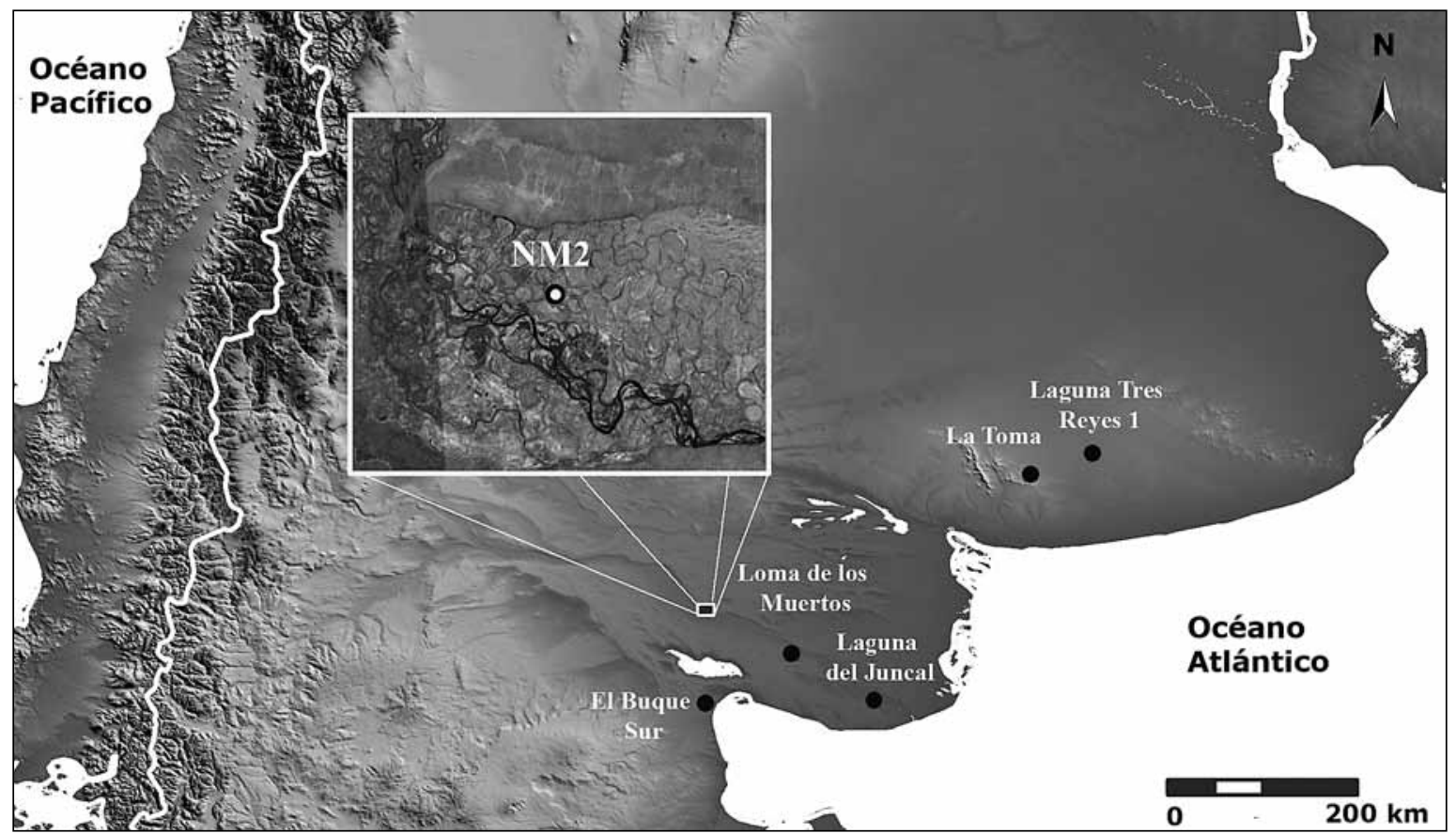

Fig. 1. Ubicación del sitio Negro Muerto 2.

Fig. 1 puede observarse la localización del sitio y la intrincada superposición de paleocauces y canales de inundación que configuran el paisaje actual.

El conjunto arqueológico de NM2 está constituido por varios esqueletos humanos, algunos restos faunísticos (restos óseos, valvas de moluscos fluviales, fragmentos de cáscara de huevo de Rheidae y escasos artefactos líticos -núcleos, desechos de talla y algunos instrumentos de molienda). Al momento del relevamiento, los materiales se encontraban dispersos en la superficie del terreno en un área aproximada de $100 \mathrm{~m}$ x $40 \mathrm{~m}$. Originalmente se disponían en una superficie elevada, posiblemente un albardón, ubicada junto al paleocauce y que fue removida por los mismos trabajos de nivelación que expusieron los restos en superficie. Durante los trabajos de campo se recuperaron los restos de varios individuos humanos cuyo análisis se presenta a continuación. Si bien también se tomaron muestras de los artefactos líticos y restos faunísticos que aparecían dispersos en la superficie del terreno, su análisis no se incluye en este trabajo. Debido al impacto diferencial de las maquinarias en los distintos sectores del sitio, los restos humanos presentan distinto grado de desarticulación y preservación. Los individuos 1 y 2 , asociados posiblemente a un mismo evento inhumatorio, fueron los menos afectados y por lo tanto pudieron ser excavados sistemáticamente. Los dos restantes, individuos 3 y 4 , presentaban un alto grado de desarticulación y fragmentación. Además de los cuatro individuos mencionados, abundantes restos humanos aparecían en superficie como producto de la dispersión ocasionada por los trabajos efectuados en el área.

\section{MATERIALES Y MÉTODOS}

Como fue señalado, los materiales analizados en este trabajo fueron recuperados en el marco de actividades de rescate arqueológico. Aunque se aplicaron técnicas sistemáticas para su excavación y muestreo, la remoción a la que fue expuesto el sitio limitó, en algunos casos, las posibilidades de obtener datos contextuales con alto grado de resolución. Para la organización de la información anatómica y con el fin de visualizar más claramente la completitud general de los conjuntos, se confeccionó un osteograma para cada individuo.

En lo que respecta a la estimación del sexo se tomaron en consideración varios criterios, con especial énfasis en las características de la pelvis y el cráneo (Brothwell 1987; Buikstra y Ubelaker 
1994; Bass 1995). En la pelvis se observaron la profundidad, ancho y simetría de la escotadura ciática; la concavidad subpúbica y rasgos de la rama isquiopúbica (Buikstra y Ubelaker 1994). En el cráneo se registraron el desarrollo de la cresta nucal, la apófisis mastoides, la glabela, el margen supraorbital y la eminencia mentoniana (Buikstra y Ubelaker 1994). También se tomaron en cuenta algunas características cuantificables del fémur (e.i. diámetro de la cabeza, ancho epicondilar y diámetros transversal y sagital del centro de la diáfisis) (Béguelin y González 2008).

En cuanto a la estimación de la edad probable de muerte se observó el grado de obliteración de las suturas craneales (Meindl y Lovejoy 1985). Complementariamente se tuvieron en cuenta las características de la sínfisis púbica (Todd 1920; Brooks y Suchey 1990), los cambios en la superficie auricular y preauricular (Lovejoy et al. 1985; Buikstra y Ubelaker 1994), la erupción dental (Ubelaker 1980) y el grado de fusión de los huesos largos (Steele y Bramblett 1988; Buikstra y Ubelaker 1994; Bass 1995; Barboza et al. 2004).

Para relevar la presencia de deformaciones artificiales del cráneo, se trabajó con técnicas de morfometría geométrica y análisis multivariados (Perez 2006b; Gómez-Valdés et al. 2007; Perez et al. 2009); sólo tres cráneos estaban en condiciones aptas para ejecutar el procedimiento mencionado. Como referencia comparativa se utilizaron, por un lado, una muestra de 112 cráneos pertenecientes a la colección Francisco Pascasio Moreno (División de Antropología del Museo de La Plata), procedentes de la laguna del Juncal, en la cuenca inferior del río Negro (Lehmann-Nitsche 1910; Perez 2006a). Por otro lado, también se consideró una muestra de siete cráneos procedentes de otros sitios arqueológicos ubicados en el valle medio e inferior del río Negro: La Victoria 5 (Prates et al. 2011), Loma de los Muertos (Prates et al. 2010 a y b) y Chimpay (Serna et al. 2009). Todos los cráneos $(\mathrm{N}=132)$ fueron posicionados según el plano de Frankfurt y fotografiados con una cámara Olympus SP 350. Se digitalizó sobre cada una de las imágenes 2D un total de 35 coordenadas cartesianas, de las cuales 4 fueron consideradas como landmarks y ubicadas en puntos craneométricos específicos (Nasion, Bregma, Lambda y Postmastoideo), y 31 como semilandmarks o coordenadas colocadas a lo largo de un contorno (programa tpsDig 2.16; Rohlf 2008). Dichas coordenadas fueron superpuestas realizando un Análisis Procrustes Generalizado (Rohlf y Slice 1990; Monteiro y dos Reis 1999; Mittereoecker y Gunz 2009) y ajustadas mediante el criterio de Bending energy (Perez et al. 2006). Con el objetivo de visualizar la información correspondiente a las morfologías de las bóvedas en forma más ordenada y eficiente, se realizó una análisis de Relative Warps o Componentes Principales con el software tpsRelw 1.49 (Rohlf 2008). En combinación con el estadístico anterior, se efectuó un análisis de Clusters Jerárquicos utilizando el software $\mathrm{R}$ 2.13.0 (RDevelopmental Team 2011, package "cluster" con método "ward"), con el propósito de conformar agrupaciones de especímenes con similitudes morfológicas. El método "ward" enfatiza y agrupa teniendo en cuenta la máxima similitud entre especímenes y, al mismo tiempo, pondera la máxima separación entre agrupaciones disímiles.

\section{RESULTADOS Y CRONOLOGÍA}

\section{Individuo 1}

El individuo 1 corresponde a un entierro primario que, si bien estaba parcialmente expuesto al momento del inicio de las actividades en el sitio, se presentaba completamente articulado y sin evidencias de remoción o desplazamiento de partes esqueletarias. Su posición era decúbito dorsal con los miembros inferiores flexionados y con orientación hacia el Este (Figura 2a). El cráneo, que reposaba sobre el parietal derecho y estaba articulado con la mandíbula, sólo presentaba incompleta la región temporal izquierda y no mostraba signos de deformación artificial. Este individuo fue determinado como un adulto medio de sexo masculino con una edad aproximada de 36-40 años al momento de la muerte. El grado de completitud del conjunto es alto (Figura 3). Se observaron escasas fracturas postdepositacionales en las costillas, las escápulas y el cráneo; y se recuperaron todos los huesos del esqueleto, con excepción de los que conforman las manos y la mayor parte de los de los pies. Teniendo en cuenta esto último se buscaron evidencias de desarticulación intencional (e. g. marcas de corte), principalmente en los sectores distales del cúbito, radio, tibia y peroné, pero no se hallaron indicadores de esta actividad. No se registraron evidencias de 


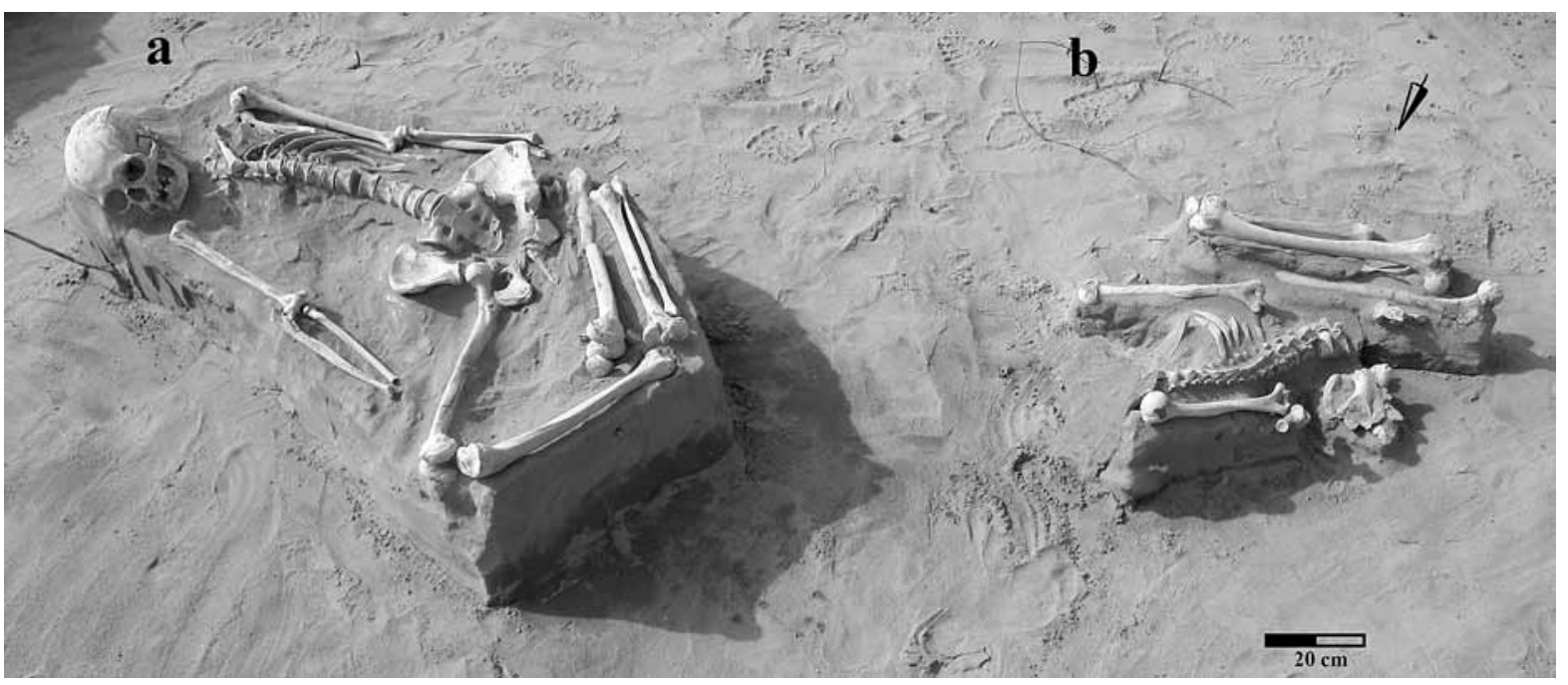

Fig. 2. Vista en planta de una de las áreas de excavación del sitio Negro Muerto 2. a) Individuo 1, b) Individuo 2.
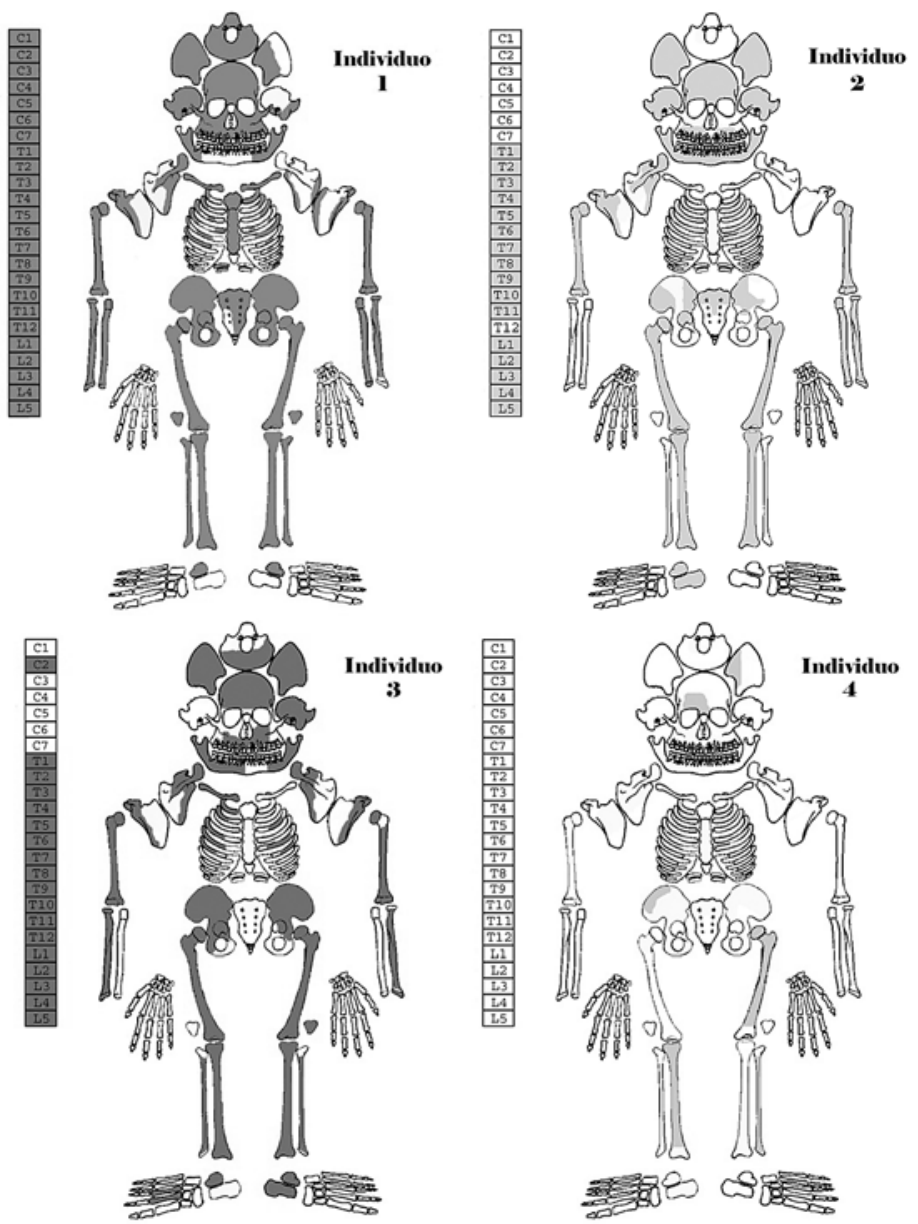

Fig. 3. En color gris, representación de elementos óseos de los cuatro individuos recuperados en Negro Muerto 2. 
meteorización en las superficies óseas ni se hallaron materiales culturales atribuibles a acompañamiento o ajuar funerario. Para determinar la cronología de este individuo se envió una muestra de costilla para su análisis radiocarbónico pero la falta de colágeno no permitió obtener ningún resultado.

Un segundo cráneo fue hallado en superficie y en asociación secundaria con los restos recién descriptos. Según el testimonio de los operarios que realizaron los hallazgos, este cráneo fue removido de entre los miembros inferiores del individuo 1 , al ser interceptado por la máquina. Esto fue constatado durante los trabajos de excavación de este individuo, cuando debajo de él se hallaron varios elementos (fragmentos de maxilar, una mandíbula y una primera vértebra toráxica) correspondientes al cráneo hallado en superficie. El grado absoluto de articulación de todo el conjunto y la elevada compactación de los sedimentos que cubrían estos elementos, indica que no fueron removidos durante las operaciones efectuadas por la actividad antrópica moderna y, por lo tanto, que conservan la posición en que fueron enterrados.

Los análisis morfológicos en dos dimensiones, efectuados en el cráneo asociado a la región pélvica del individuo 1 , muestran que la bóveda fue deformada artificialmente. Esto permite agruparlo por afinidad junto con los cráneos que presentan una forma comprimida en sentido fronto-occipital y una proyección de la zona lámbdica. Si bien el cluster o agrupamiento es claro, la información generada y disponible en 2D, no permite asignar este cráneo en particular a una de las categorías morfológicas de los tipos de deformación definidas inicialmente por Imbelloni (1924-25), debido a que el espécimen en cuestión se ubica en una zona intermedia entre dos tipos (Figura 4). En otras palabras, los análisis de morfometría geométrica han permitido reconocer la presencia de una alteración artificial en la bóveda, aunque no es posible a través de ellos, la asignación a uno de los tipos discretos propuestos con anterioridad en forma terminante. Aún así, desde la visualización en un plano bidimensional puede señalarse que la deformación sólo podría corresponder al tipo circular o al tipo tabular oblicuo, quedando descartados los tipos tabular erecto planolámbdico y planofrontal (Imbelloni 1924-25; Dembo e Imbelloni 1938).

\section{Individuo 2}

El individuo 2 también corresponde a un entierro primario, ubicado $75 \mathrm{~cm}$ al este del individuo 1 (Figura 2b). Al momento de comenzar los trabajos en el campo sólo se encontraba expuesta una porción del fémur derecho. Aunque las superficies óseas no mostraban marcas de meteorización, el esqueleto exhibía mayores efectos de remoción que el individuo 1. Los restos estaban completamente articulados, con excepción de algunos huesos largos, y se encontraban orientados en dirección noreste con los miembros inferiores flexionados sobre su lateral izquierdo. Los miembros superiores, también flexionados, y la caja toráxica se encontraban dirigidos al suelo. Fue definido como un adulto joven de sexo masculino con una edad aproximada de 30-35 años al momento de la muerte. Si bien presenta un grado de completitud $e$ integridad alto, es notable la ausencia de algunos elementos óseos, tales como: el cráneo, algunas vertebras y algunos huesos largos (radio y cúbito derechos y peroné izquierdo). Nuevamente no se recuperaron los huesos que conforman los pies y las manos. Tampoco se hallaron las vértebras cervicales ni la primera vértebra toráxica, pese a que el resto de la columna se hallaba perfectamente articulada. Sólo se recuperó la escápula derecha, aunque con el sector del cuerpo muy deteriorado (Figura 3). Al igual que con el individuo 1 , no se encontró ningún tipo de material arqueológico que pueda considerarse como acompañamiento o ajuar funerario. Para este esqueleto se obtuvo una edad radiocarbónica, a partir de una muestra costilla, de $1.586 \pm 47$ años AP (AA89359) (Tabla 1).

Tabla 1. Fechas radiocarbónicas de los esqueletos de Negro Muerto 2 (todos los fechados fueron realizados en Accelerator Mass Spectrometry Laboratory, University of Arizona).

\begin{tabular}{|c|c|c|c|c|c|c|}
\hline Código \# & Muestra ID & $\begin{array}{l}\text { Material } \\
\text { datado }\end{array}$ & ${ }^{13} \mathrm{C}$ & años ${ }^{14} \mathrm{C}$ AP & $\begin{array}{l}\text { Edad Calibrada (años } \\
\text { AD) }\end{array}$ & Deformación craneana \\
\hline- & E1 & - & - & - & - & No presenta \\
\hline AA89359 & E2 & Costilla & -19.9 & $1.586 \pm 47$ & $540-633$ & Circular/Tabular oblicua \\
\hline AA91545 & E3 & Costilla & -20 & $1.637 \pm 48$ & $428-536$ & No presenta \\
\hline AA91546 & E4 & Costilla & -18.7 & $1.283 \pm 44$ & $768-873$ & - \\
\hline
\end{tabular}




\section{Individuo 3}

El individuo 3 fue hallado en una posición parcialmente superficial, con un avanzado grado de desarticulación producto de la remoción generada durante la nivelación del terreno, $48 \mathrm{~m}$ al sur de los individuos 1 y 2 . Sólo estaban articulados los pocos elementos que se mantuvieron enterrados, sobre todo los correspondientes a las regiones toráxica y lumbar de la columna vertebral, y parte de los miembros inferiores; esto permitió inferir que se trataba de un probable entierro del tipo primario. Al igual que en los casos anteriores no se hallaron el tarso, el carpo ni las falanges. Las escápulas se encontraban incompletas y algunos huesos largos presentaban las epífisis sin fusionar, mientras que los mismos huesos de distinta lateralidad sí presentaban fusión (Figura 3). El cráneo también se encontró con un alto grado de fragmentación y no presenta evidencias de deformación artificial. Para este individuo, de sexo masculino, se estimó una edad de entre 17 y 25 años al momento de su muerte. Sólo algunos elementos presentaban evidencias de meteorización sobre sus superficies (entre los estadios 2 y 3, sensu Behrensmeyer 1987), principalmente los huesos largos de los miembros superiores, los cuales se encontraban expuestos al momento del hallazgo. No se encontró ningún tipo de acompañamiento o ajuar funerario asociado a los restos humanos. A partir de un fragmento de costilla se obtuvo una cronología absoluta de $1.637 \pm 48$ años ${ }^{14} \mathrm{C}$ AP (AA91545) (Tabla 1).
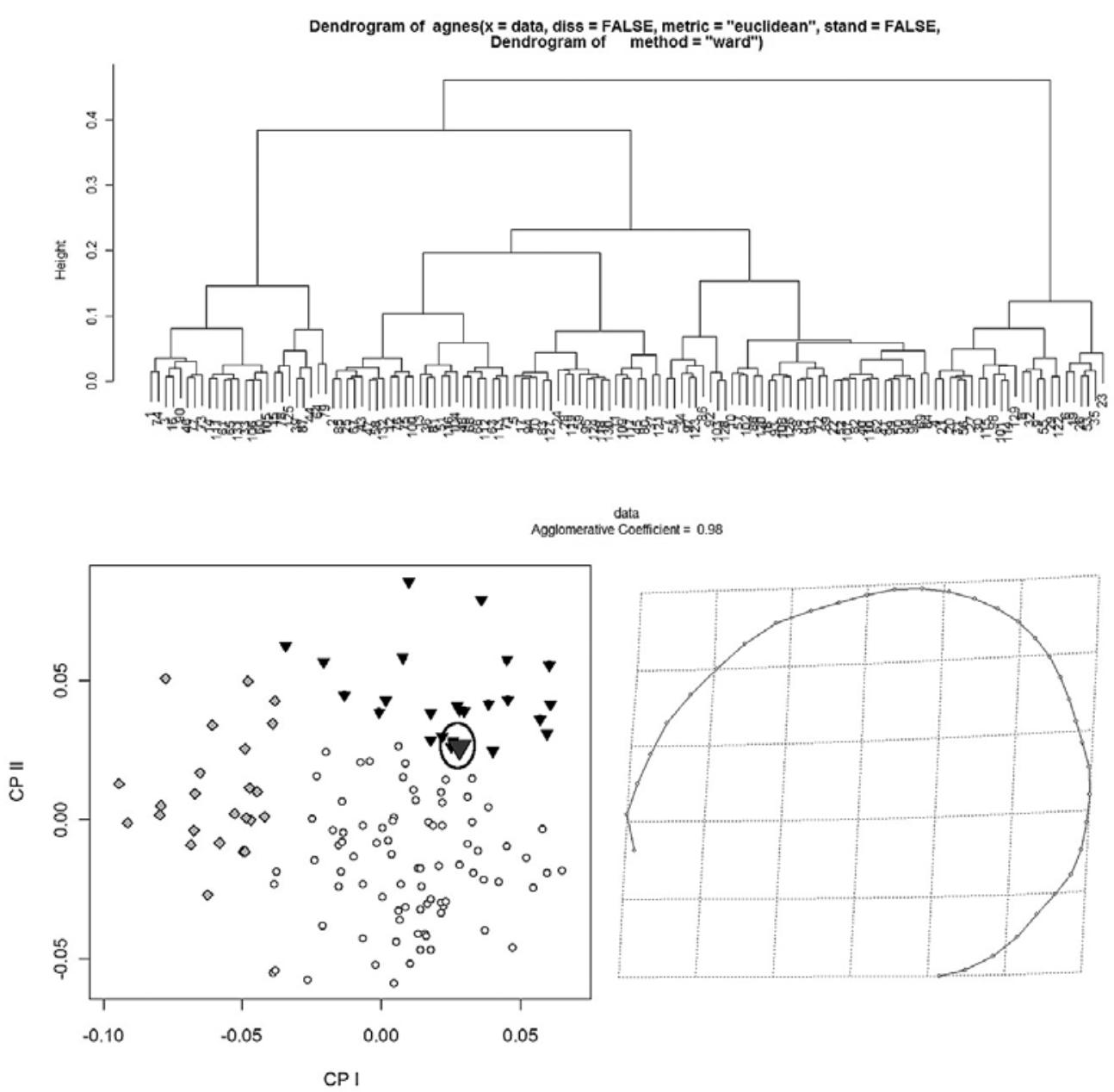

Fig. 4. Dendrograma con método ward exhibiendo los clusters formados sobre la muestra. Gráfico de Componentes Principales reflejando los clusters. Grilla de deformación correspondiente a la morfología del cráneo del individuo 2. 


\section{Individuo 4}

Por último, el individuo 4 fue hallado $50 \mathrm{~m}$ al suroeste del individuo 3 . Todos los huesos se encontraban en la superficie del terreno y, a diferencia del anterior, completamente desarticulados. La completitud general de este individuo fue la más pobre de la muestra y la mayor parte de los elementos mostraba algún grado de meteorización (estadio 3, sensu Behrensmeyer 1987). El conjunto está representado por unos pocos elementos del cráneo y de los miembros inferiores (Figura 3). Sobre la base de algunas variables métricas tomadas sobre el fémur se determinó que corresponde a un individuo de sexo masculino. La edad al momento de la muerte y la presencia de deformación artificial del cráneo no pudieron ser determinadas debido al alto grado de fragmentación que presentan los huesos y a la ausencia de estructuras diagnósticas. Como en los casos anteriores, no se hallaron otros materiales culturales que puedan interpretarse como ajuar o acompañamiento funerario. Un fechado radiocarbónico sobre un fragmento de costilla arrojó una

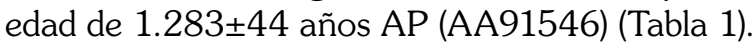

\section{DISCUSIÓN}

El sitio NM2 está emplazado sobre una elevación del terreno ubicada sobre un paleocauce. Las diferentes fechas obtenidas para las muestras sugieren que el mismo sitio fue utilizado recurrentemente para prácticas mortuorias por un lapso de al menos 400 años, entre los 1.600 y 1.200 años AP. Los trabajos realizados en el sitio, muestran que los efectos de las actividades agrícolas removieron numerosos (al menos 10) esqueletos humanos; algunos fueron muy afectados y dispersados en la superficie del terreno $y$ otros, como los analizados en este trabajo, se conservaron con mayores grados de completitud e integridad. Los individuos 1 y 2 exhiben un grado de alteración bajo, conservando buena parte de los elementos óseos articulados; sobre todo el primero, en el que no se observaron indicadores de acción mecánica postdepositacional. Tampoco se registraron en ellos evidencias de meteorización en las superficies óseas, lo que muestra que su exposición aérea fue muy breve o nula. La desarticulación y/o pérdida de algunos huesos largos del individuo 2 , atribuidas a la remoción reciente del depósito en que se encontraba, no afectó de manera significativa la completitud del esqueleto ni la posición anatómica en que fue inhumado. A diferencia de los conjuntos 1 y 2 , los 3 y 4 sufrieron un efecto mecánico postdepositacional severo. El primero de estos últimos, el conjunto 3 , presenta un alto grado de desarticulación y desplazamiento de partes esqueletarias aunque se preservaron articulados los elementos de la columna vertebral. De los individuos analizados, el 4 es el que mayor acción mecánica sufrió luego de su entierro. No conserva elementos articulados, presenta una fracturación alta y un grado de completitud bajo. El registro de evidencias de meteorización de las superficies óseas, en la mayor parte de los elementos (estadio 3, sensu Behrensmeyer 1978), demuestra que éstos estuvieron expuestos en superficie un tiempo relativamente prolongado.

Respecto a la completitud general de cada individuo, es posible observar un patrón en la ausencia y preservación de partes esqueléticas. En el caso del esqueleto axial, se observa que las vértebras cervicales y el esternón son los elementos menos frecuentes. Aunque las costillas están generalmente presentes, aparecen siempre con un alto grado de fracturación. Los cráneos de todos los individuos también aparecen fragmentados; en todos los casos por la actividad antrópica reciente. En cuanto al esqueleto apendicular, en todos los casos las escápulas se encuentran dañadas en el área del cuerpo. En los cuatro individuos faltan todos los huesos de las manos y están escasamente representados los de los pies. Si bien la acción antrópica reciente pudo ser responsable de este sesgo en los individuos 3 y 4 , no sería explicativa para los individuos 1 y 2 , que se hallaban in situ y no registraban prácticamente evidencias de remoción. Aunque ciertos elementos de pequeño tamaño (e. g. falanges, tarsos y carpos) son más susceptibles de migrar por la acción de roedores (e.g. González 2007), no se registraron en el sitio indicadores claros de este tipo de agente. Y como tampoco se observaron evidencias de desarticulación intencional de los sectores distales de los miembros, este sesgo en la representación esqueletaria no puede ser resuelto con la información disponible hasta el momento.

Otro aspecto tafonómico relevante de este contexto mortuorio es la presencia de un segundo cráneo asociado a los miembros inferiores del individuo 1 y la ausencia del correspondiente al individuo 2 . Teniendo en cuenta que la primera vértebra toráxica 
Tabla 2. Fechas radiocarbónicas de otros sitios con deformación Tabular oblicua.

\begin{tabular}{|l|l|l|l|c|}
\hline \multicolumn{1}{|c|}{ Sitio } & \multicolumn{1}{|c|}{ Código ${ }^{*}$} & \multicolumn{1}{|c|}{ Muestra ID } & años ${ }^{14}$ C AP & $\begin{array}{c}\text { Edad Calibrada } \\
\text { (años AD) }\end{array}$ \\
\hline \multirow{2}{*}{ Laguna Tres Reyes $1^{1}$} & AA24048 & TR1-1 & $2.245 \pm 55$ & $2.350-2.120$ \\
& AA24048 & TR1-10 & $2.470 \pm 60$ & $2.750-2.350$ \\
\hline La Toma & SI-6475 & & $2.075 \pm 70$ & $1.886-2.060$ \\
\hline Loma de los Muertos & AA81827 & LM1.E1.1 & $2.088 \pm 46$ & $1.882-2.121$ \\
\hline Laguna del Juncal & AA72632 & RN 3 & $2.600 \pm 47$ & $2.370-2.759$ \\
\hline El Buque Sur & AA72634 & RN 4 & $2.642 \pm 47$ & $2.488-2.788$ \\
\hline El Buque Sur & AA70720 & BS I & $2.195 \pm 49$ & $2.009-2.019$ \\
\hline
\end{tabular}

(hallada in situ debajo del individuo 1) articula con la columna del individuo 2 y completa su serie de vértebras toráxicas, es razonable plantear que el cráneo de este último es el que se encontró asociado secundariamente al primero. Es decir, que el cráneo del individuo 2 fue separado del resto del cuerpo y enterrado entre los miembros inferiores del individuo 1. En primer lugar, esto implica que la porción superior del esqueleto axial (neurocráneo, mandíbula y, al menos, una parte de la porción toráxica de la columna) del individuo 2 fue desarticulada y asociada al individuo 1 ; y en segundo lugar, que el contexto mortuorio integrado por estos individuos constituye un entierro primario doble. Asumiendo la contemporaneidad de los eventos de entierro de ambos individuos, puede inferirse para el conjunto 1 , una cronología similar a la del 2 , es decir de alrededor de 1.600 años AP.

Un tercer aspecto destacable del sitio NM2 es que el tipo de deformación artificial registrado en el cráneo del individuo 2 amplía el rango cronológico de las deformaciones cefálicas, surgido de los datos disponibles hasta ahora para el norte de la región patagónica y sur de la región pampeana. El esquema cronológico de las deformaciones fue planteado inicialmente por Bórmida (1950, 1953-54) en forma relativa, y precisado más recientemente con fechados radiocarbónicos (e. g. Berón y Baffi 2003; Bernal et al. 2008; Berón y Luna 2009; Perez et al. 2009). Los distintos modelos propuestos, aunque exhiben pequeñas variaciones, se comportan de manera similar: las morfologías aplanadas fronto-occipitalmente con proyección en la zona lámbdica (tipo circular, Imbelloni 1924-25; Dembo e Imbelloni 1938) se ubican cronológicamente en el Holoceno medio-tardío, con fechados anteriores a 3.000 años AP. Las morfologías fuertemente aplanadas en la zona lámbdica con expansión del hueso frontal (tipo tabular erecto planolámbdico, Imbelloni 1924-25; Dembo e Imbelloni 1938) son asignables al Holoceno tardío final, con fechados de entre 1.500 y 400 años AP. La morfología aplanada fronto-occipitalmente en forma oblicua (tipo tabular oblicua, Imbelloni 1924-25, Dembo e Imbelloni 1938) ha sido asignada al período de ca. 2.600-2.000 años $\mathrm{AP}$ (para mayor detalle véase discusión en Perez et al. 2009). Como hemos señalado, los estudios de morfometría geométrica y estadística multivariada efectuados sobre el espécimen mencionado, indican que se trata necesariamente de la variante circular o tabular oblicua. Para la primera, se disponía hasta ahora de fechados previos a los 3.000 años AP (Beguelin et al. 2006; Bernal et al. 2008), y de alrededor de 2.000 años AP para la segunda (e. g. Bernal et al. 2008; Prates et al. 2010a). Esto implica que el tipo de deformación registrado en el individuo 2 sería 1.500 o 500 años más tardía que lo esperado considerando el esquema cronológico actual, según corresponda al tipo circular o tabular oblicuo, respectivamente. Sobre la base de la proximidad entre la cronología de ambos tipos de deformación y los $1.586 \pm 47$ años AP obtenidos para el conjunto, la hipótesis más conservadora sería que la deformación de este último se ajuste al tipo tabular oblicuo. Para esta variante deformatoria se dispone de unos pocos fechados radiocarbónicos de sitios arqueológicos de Pampa y Patagonia: Laguna Tres Reyes 1 y La Toma (en la región pampeana)

Las dataciones fueron realizadas sobre individuos que no presentaban la deformación Tabular oblicua pero que se encontraban formando parte de la misma inhumación que los deformados, por lo que deben considerarse en forma tentativa. 
(Madrid y Politis 1991; Madrid y Barrientos 2000); Loma de los Muertos y Laguna del Juncal (cuenca del río Negro) (Bernal et al. 2008; Prates et al. 2010a) y El Buque Sur (Golfo San Matías) (Favier Dubois et al. 2009); todos ubicados alrededor del período comprendido entre los 2.600 y 2.000 años AP (Tabla 2). La nueva fecha relativamente tardía de NM2, sin embargo, se aproxima bastante a las obtenidas para cráneos con el mismo tipo de deformación procedentes del sitio Cañada Seca (Mendoza): 1.790 \pm 50 y $1.420 \pm 60$ años AP (Beguelín et al. 2006).

\section{CONSIDERACIONES FINALES}

Como ya se ha mencionado en este trabajo, los sitios arqueológicos con presencia de restos óseos humanos son numerosos en el valle del río Negro. Sin embargo, la información cronológica y contextual obtenida de ellos desde finales del siglo XIX es escasa y poco sistemática. En este sentido, el sitio Negro Muerto 2 provee información novedosa y precisa sobre las prácticas mortuorias en el sector medio de la cuenca durante el Holoceno tardío. Un aspecto interesante de NM 2 es que muestra tendencias similares a las observadas en otros sitios localizados en otros sectores del mismo valle (Prates et al. 2010 a y b; Prates et al. 2011). Sobre todo, en cuanto a la recurrencia en la ubicación de las áreas de entierro en superficies elevadas asociadas a paleocauces del río y en cuanto al registro de evidencias de actividades domésticas en las mismas áreas donde se efectuaron las prácticas mortuorias. $\mathrm{Si}$ bien no se presentaron aquí los resultados del estudio de los materiales líticos y faunísticos del sitio, la presencia de este tipo de registro a lo largo de la mayor parte del área de dispersión de restos humanos, sugiere la superposición espacial (aunque no necesariamente temporal) de áreas domésticas y mortuorias. Desde el punto de vista cronológico, las evidencias de NM2 muestran que el mismo espacio fue ocupado de manera recurrente, al menos entre los 1.600 y 1.200 años AP.

Además de los procesos postdepositacionales que afectaron la integridad del sitio, se reconoció la manipulación postmortem de uno de los cuerpos. El cráneo y otras piezas óseas del individuo 2 fueron desarticulados y enterrados junto con el 1 . Aunque la compleja práctica de redisponer partes anatómicas (disposiciones sensu Berón et al. 2002) no había sido registrada hasta ahora en la cuenca del río Negro, ya ha sido descripta para el Holoceno tardío en algunos sitios del norte de Patagonia y del centro-sur de la región pampeana (Mazzia et al. 2004; Martínez et al. 2006; Berón y Luna 2007).

Por último, aún reconociendo las limitaciones que implica asignar la deformación artificial de un cráneo a una categoría discreta, debido a que la variación entre ellas es de carácter continuo (Perez 2007), los análisis morfométricos en 2 D efectuados sobre el cráneo del individuo 2 permiten atribuirla a una deformación circular o tabular oblicua. Por afinidad cronológica, este cráneo se ubica más cerca de la segunda de las variantes, pero con los análisis efectuados no es posible validar esta posibilidad taxativamente. Probablemente la aplicación de técnicas morfogeométricas en 3D proporcione el grado de resolución necesario para resolver el problema. En definitiva, sea circular o tabular oblicuo el tipo de deformación registrado en NM2, implica una variación de su esquema cronológico para Pampa y Patagonia.

\section{AGRADECIMIENTOS}

Queremos expresar nuestro agradecimiento a S. Iván Perez por su constante colaboración durante el estudio de los materiales del sitio y por la lectura de versiones preliminares del artículo. A Daniel Cabaza por la oportuna denuncia de los hallazgos y por el apoyo recibido desde el Museo Paleontológico Municipal de Lamarque (Río Negro). Los trabajos fueron realizados con recursos financieros obtenidos del proyecto PIP-CONICET № 338/10.

\section{BIBLIOGRAFÍA}

BARBOZA, C., M. A. BORDACH y O. MENDONCA. 2004. Osteología Humana. Determinación de la edad y el sexo. El sitio SJ TIL 43. Universidad Nacional de Río Cuarto, Río Cuarto.

BASS, W.M. 1995. Human Osteology: A Laboratory and Field Manual of the Human Skeleton. Special Publication, Missouri Archaeological Society. Columbia.

BÉGUELIN, M., V. BERNAL, M. DEL PAPA, P. NOVELLINO y G.BARRIENTOS. 2006. El poblamiento humano tardío del sur de Mendoza y su relación con el norte de Patagonia: una discusión bioarqueológica. Anales de Arqueología y Etnología 61: 5-25. 
BÉGUELIN, M. y P. N. GONZALEZ. 2008. Estimación del sexo en poblaciones del sur de Sudamérica mediante funciones discriminantes para el fémur. Revista Argentina de Antropología Biológica 10(2): 55-70.

BEHRENSMEYER, A. K. 1978. Taphonomic and ecological information from bone weathering. Paleobiology 4(2):150-162.

BERNAL, V., P.N. GONZALEZ, S.I. PEREZ y H.M. PUCCIARELLI. 2008. Entierros humanos del noreste de Patagonia: nuevos fechados radiocarbónicos. Magallania 36(2):175-183.

BERÓN, M. y L. LUNA. 2007. Modalidades de entierro en el sitio Chenque I. Diversidad y complejidad de los patrones mortuorios de los cazadores-recolectores pampeanos. En: Arqueología en las Pampas, editado por Bayón, C. I. González, N. Flegenheimer, A. Pupio y M. Frère, pp. 129-142. Sociedad Argentina de Antropología. Buenos Aires.

2009. Distribución espacial y cronológica de la deformación craneana tabular erecta en Pampa y Norpatagonia. En: Arqueología de la Patagonia. Una Mirada desde el Último Confín, compilado por M. Salemme, F. Santiago, M. Álvarez, E. Piana, M. Vázquez y M. E. Mansur, pp. 561-575. Editorial Utopías, Ushuaia.

BERÓN, M., E. BAFFI, R. MOLINARI, C. ARANDA, L. LUNA y A. CIMINO. 2002. El chenque de Lihué Calel. Una estructura funeraria en las "Sierras de la Vida". En: Del Mar a los Salitrales. 10.000 de Historia Pampeana en el Umbral del Tercer Milenio, editado por Mazzanti D. L., M. A. Berón y F. W. Oliva, pp. 87. Laboratorio de Arqueología, Facultad de Humanidades, UNMdP, Mar del Plata.

BERÓN, M.A. y E.I. BAFFI. 2003. Procesos de cambio cultural en los cazadores-recolectores de la provincia de La Pampa, Argentina. Intersecciones en Antropología 4: 29-43.

BORMIDA, M. 1950. Cementerios indígenas prehispánicos en la zona de la Laguna del Juncal. Anales del Museo Nahuel Huapi Perito Dr. Francisco P. Moreno 2: 101-108. 1953-54. Los antiguos Patagones. Estudio de craneología. Runa VI (1-2): 55-96.

BROOKS, S. y J. SUCHEY. 1990. Skeletal age determination based on the Os Pubis: a comparison of the AcsadiNemeskeri and Suchey-Brooks methods. Human Evolution 5:227-238.

BROTHWELL, D.R. 1987. Desenterrando Huesos. La excavación, Tratamiento y Estudio de Restos del Esqueleto Humano. Fondo Cultura Económica, México.

BUIKSTRA J. y D. UBELAKER. 1994. Standards for data collection from human skeletal remains. Arkansas Archaeological Survey Research Series 44, Arkansas.
CABRERA, A. 1976. Regiones Fitogeográficas Argentinas. Enciclopedia Argentina de Agricultura y Jardinería, T II. Editorial Acme. Buenos Aires.

COCILOVO, J. A. y R. GUICHÓN. 1994. La deformación craneana "pseudocircular" en el grupo prehistórico de Laguna del Juncal. Revista Internacional de Biología de Poblaciones 2(2):13-28.

COLANTONIO, S. y A. MARCELINO. 1982. Nueva contribución al conocimiento antropológico de los restos aborígenes de San Blas e isla Gama (pcia. de Buenos Aires). Publicaciones del Instituto de Antropología 38-39: 97-153. 1983. Relaciones morfológicas de los aborígenes prehispánicos del territorio argentino: San Blas e isla Gama. Publicaciones del Instituto de Antropología 41: 51-66.

DEMBO, A. y J. IMBELLONI. 1938. Deformaciones intencionales del cráneo de carácter étnico. J. Anesi. Buenos aires.

FAVIER DUBOIS, C. M., F. BORELLA y R. H. TYKOT. 2009. Explorando tendencias en el uso humano del espacio $y$ los recursos en el litoral rionegrino (argentina) durante el Holoceno medio y tardío. En: Arqueología de la Patagonia. Una Mirada desde el Último Confín, compilado por M. Salemme, F. Santiago, M. Álvarez, E. Piana, M. Vázquez y M. E. Mansur, pp. 985-997. Editorial Utopías, Ushuaia.

FISHER, A. y L. R. NACUZZI. 1992. La destrucción sistemática del paisaje y de los sitios arqueológicos. El caso del Valle de Viedma. Arqueología 2: 189-229.

GOMEZ-VALDES, J.A., J.B. MARTINEZ y A.R. PACHECO. 2007. Morfometría geométrica aplicada al estudio De la deformación cefálica intencional. Estudios de Antropología Biológica XIII: 117-134.

GONZÁLEZ, M. E. 2007. Estudios de interés tafonómico en los restos óseos humanos de la laguna Tres Reyes 1 (Partido de Adolfo Gonzales Chaves). Intersecciones en Antropología 8: 215-233.

GORDÓN, F. y G. GHIDINI. 2007. Análisis bioarqueológico de la violencia interpersonal. El valle inferior del río Negro (República Argentina) durante el Holoceno tardío. Revista Werken 9:27-45.

HAUMAN-MERCK, L. 1913. Étude phytogéographique de la región du Rio Negro inférieur (Republique Argentine). Anales del Museo Nacional de Historia Natural 24: 289-444.

IMBELLONI, J. 1924-25. Deformaciones intencionales del cráneo en Sud América. Revista del Museo de La Plata 28: 329-407.

LEHMANN-NISTCHE, R. 1926. El revestimiento con ocre rojo de las tumbas prehistóricas y su significado. Physis 8: 390-396. 
LEHMANN-NITSCHE, R. 1930. Un cráneo patagón con pinturas geométricas en rojo y negro procedente de San Blas (costa atlántica). Revista del Museo de la Plata 32: 239-280.

LISTA, R. 1879. Viaje al país de los tehuelches: exploraciones en la Patagonia austral. Imprenta Martín Biedma, Buenos Aires.

1880. Mis exploraciones y descubrimientos en la Patagonia, 1877-1880. Imprenta Martín Biedma, Buenos Aires.

LOVEJOY, O., R. MEINDL, T. PRYZBECK y R. MENSFORTH.1985. Chronological methamorphosis of the auricular surface of the ilium: a new method for the determination of adult skeletal age at death. American Journal of Physical Anthropology 68: 15-28.

LUCHSINGER, H. 2006. Estratigrafía de los sitios Negro Muerto y Angostura 1. Laboratorio 3, Departamento Científico de Arqueología, Facultad de Ciencias Naturales y Museo, Universidad Nacional de La Plata, La Plata. MS.

MADRID, P. y G. BARRIENTOS. 2000. La estructura del registro arqueológico del sitio Laguna Tres Reyes 1 (provincia de Buenos Aires): nuevos datos para la interpretación del poblamiento humano en el sudeste de la región pampeana a inicios del Holoceno tardío. Relaciones de la Sociedad Argentina de Antropología XXV: 179-206.

MADRID, P. y G. POLITIS. 1991. Estudios paleoambientales en la Región Pampeana: un enfoque multidisciplinario del sitio La Toma. Actas del XI Congreso Nacional de Arqueología Chilena I: 131-152.

MARTÍNEZ, G. y M. J: FIGUERERO TORRES. 2000. Sitio arqueológico La Petrona (Partido de Villarino, Provincia de Buenos Aires). Relaciones 25: 227-247.

MARTÍNEZ, G., P. BAYALA, G. FLENSBORG y R. LÓPEZ. 2006. Análisis preliminar de los entierros humanos del sitio Paso Alsina 1 (partido de Patagones, provincia de Buenos Ares). Intersecciones en Antropología 7: 95-108.

MAZZIA, N., C. SCABUZZO y R. GUICHÓN. 2004. Sobre cráneos, pelvis y otros huesos. Entierros humanos en el sitio El Guanaco. En: Aproximaciones contemporáneas a la arqueología pampeana. Perspectivas teóricas, metodológicas, analíticas y casos de estudio, editado por Martínez, G., M. Gutiérrez, R. Curtoni, M. Berón y P. Madrid, pp.293-304. Facultad de Ciencias Sociales, Universidad del Centro de la Provincia de Buenos Aires. Buenos Aires. Olavarría.

MEINDL, R.S. y C.O. LOVEJOY. 1985. Ectocranial Suture Closure: a revised method for the determination of skeletal age at death based on the lateral-anterior sutures. American Journal of Physical Anthropology 68(1):57-66.
MITTEROECKER P. y P. GUNZ. 2009. Advances in Geometric Morphometrics. Evol Biol 36:235-247.

MOLDES DE ENTRAIGAS, B. 1983. Arqueología y Etnohistoria del bajo curso del río Negro. Presencia hispánica en la Arqueología Argentina 2: 877-893.

MONTEIRO, L. y S. FURTADO dos REIS. 1999. Principios de morfometría geométrica. Holos Editora. Ribeirao Preto.

MORENO, F. P. 1874. Description des cimetières et paraderos prehistoriques de patagognie. Revue d'Antropologie 3: 72-90.

MUSTERS, G. C. (1997[1869-1970]). Vida entre los patagones. El Elefante Blanco, Buenos Aires.

OUTES, F. F. 1926. Noticias sobre los resultados de mis investigaciones antropológicas en la extremidad sudeste de la provincia de Buenos Aires. Physis 8: 387-390.

PEREZ, S.I. 2006a. El poblamiento holocénico del Sudeste de la Región Pampeana: un estudio de morfometría geométrica craneofacial. Tesis doctoral. Universidad Nacional de La Plata, La Plata. MS.

2006b. Influencia de la deformación artificial del cráneo sobre la morfología facial: implicancias para los estudios de relaciones evolutivas inter-poblacionales. Revista Argentina de Antropología Biológica 8(1): 1-27.

2007. Artificial cranial deformation in South America: a geometric morphometrics approximation. Journal of Archaeological Science 34: 1649-1658.

PEREZ, S.I., C. DELLA NEGRA, P. NOVELLINO, P.N. GONZALEZ, V. BERNAL, E. CUNEO y A. HAJDUK. 2009. Deformaciones artificiales del cráneo en cazadoresrecolectores del Holoceno medio-tardío del noroeste de Patagonia. Magallania 37(2): 77-90.

PEREZ, S.I., V. BERNAL, y P.N. GONZALEZ. 2006. Differences between sliding semi-landmark methods in geometric morphometrics, with an application to human craniofacial and dental variation. Journal of Anatomy 208:769-784.

PRATES, L. 2008. Los indígenas del río Negro. Un enfoque arqueológico. Colección Tesis doctorales de la Sociedad Argentina de Antropología, Buenos Aires.

PRATES, L., G. A. FLENSBORG y P. BAYALA. 2010a. Caracterización de los entierros humanos del sitio Loma de Los Muertos (valle medio del río Negro, Argentina). Magallania 38(1): 147-162.

PRATES, L., V. DI PRADO, E. MANGE y A. SERNA. 2010b. Sitio arqueológico Loma de los Muertos. Múltiples ocupaciones sobre un médano del este de Norpatagonia (Río Negro, Argentina). Magallania 38(1):163-179.

PRATES, L., H. LUCHSINGER, C. SCABUZZO y D. MANSEGOSA. 2011. Investigaciones arqueológicas en el sitio 
La Victoria 5 (Departamento de General Conesa, Río Negro). Intersecciones en Antropología 12: 109-120.

ROHLF, F.J y D.E. SLICE. 1990. Extensions of the Procrustes Method for the optimal superimposition of landmarks. Systematic Zoology 39:40-59.

ROHLF, F.J. 2008. tps serie softwares. Disponible en http// life.bio.sunysb.edu/morph/.

SANGUINETTI DE BÓRMIDA, A. C. 1999. Proyecto Norpatagonia. Arqueología de la Costa Septentrional. Separata de Anales de la Academia Nacional de Ciencias de Buenos Aires, Buenos Aires.

SERNA A., P. GONZALEZ, S. I. PEREZ y L. PRATES. 2009. Análisis bioarqueológico del sitio Chimpay (Río Negro). Trabajo presentado en IX Jornadas Nacionales de Antropología Biológica. Puerto Madryn, Provincia de Chubut, 20 al 23 de octubre de 2009.

STEELE, D.G. y C.A. BRAMBLETT. 1988. The anatomy and biology of the human skeletal. Texas A\&M University Press. College Station.

STROBEL, P. 1867. Paradero prehistoric in Patagonia. Atti della società Italiana de Scienze Naturali 10: 167-171. 1868. Materiale di palaetnologia comparata, racolti in Sud-américa. Dalla tipografía di G. Ferrari, Parma.
TODD, T. 1920. Age Changes in the Pubic Bone. I. The male White Pubic. American Journal of Physical Anthropology 3 (3): 285-335.

UBELAKER, D. 1980. Human Skeletal Remains. Excavation, Analysis, Interpretation, Smithsonian Institution. Washington.

VERNEAU, R. 1903. Les anciens patagons. Contribution a l'etude des races précolombienes de l'Amerique du Sud. Impr. de Mónaco, Mónaco.

VERNEAU, R. y H. de la VAULX. 1900. Les anciens habitants des rives du Colhue-Huapi (Patagonie). En : Actes du Congrès International des Americanistes, XII e Session, pp. 135-137. Paris.

VIGNATI, M. A. 1923. Las llamadas hachas patagónicas. Descripción de ejemplares y nueva interpretación. Comunicaciones del Museo Nacional de Historia Natural 2 (3): 17-44.

1931. Investigaciones antropológicas en el litoral marítimo suratlántico bonaerense. Notas Preliminares del Museo de La Plata 1: 19-31.

1937. Origen étnico de los cráneos pintados de San Blas. Relaciones 1: 52-57. 
